\title{
Diversidad cultural y diálogo intercultural en la ciudad. El caso de Mångkulturellt centrum en el municipio de Botkyrka, Suecia
}

\section{Cultural diversity and intercultural dialogue in the city. The case study of Mångkulturellt centrum in the municipality of Botkyrka, Sweden}

\author{
Mónica Riaza de los Mozos ${ }^{1}$ \\ ${ }^{1}$ Candidata a Doctora en Gestión Cultural - Cooperación Cultural y \\ Desarrollo, Universitat de Girona, Gerona, España.
} Correo electrónico: riazadm@yahoo.com

(C) Revista Internacional de Estudios Migratorios. CEMyRI. UAL (España) 


\section{Resumen}

Introducción: El mundo globalizado ha facilitado la proliferación de los contactos entre las culturas. Ello ha conllevado transformaciones culturales y sociales, a menudo resultando en profundas divergencias sociales. Ante este panorama cambiante, un nuevo debate desafía las políticas públicas. Y el poder de la cultura como requisito de la paz, fuente de bienestar y como recurso para el desarrollo sostenible es más importante que nunca.

Con el fin de conocer la potencialidad de las políticas culturales en el territorio teniendo como base un contexto asentado en la diversidad cultural y el diálogo intercultural, la presente investigación se centra en las políticas culturales elaboradas en el municipio de Botkyrka, al sur de la provincia de Estocolmo (Suecia), a través del estudio del caso de la fundación Mångkulturellt centrum.

Método: El método cualitativo de la investigación es el aplicado en el estudio, desarrollando un trabajo descriptivo y exploratorio, donde la teoría y la lógica están unidas. A través del paradigma interpretativo hemos conseguido realizar el estudio de un contexto particular y enfatizar la comprensión de la experiencia humana. Las tres técnicas aplicadas son el estudio de caso, el análisis de contenido y entrevistas focalizadas (semiestructuradas) a expertos del propio centro de estudio del caso.

Resultados: El caso de Mångkulturellt centrum permite estudiar el análisis detallado de la fundación como proyecto que mejora la diversidad cultural y el diálogo intercultural en el territorio, identificamos acciones concretas aplicadas y exitosas, y detectamos desafíos a los que se enfrentan las políticas locales en la actualidad.

Conclusión: La investigación corrobora la importancia de tener organizaciones públicas que logren competencias interculturales, se abran al diálogo intercultural y trabajen junto con los propios inmigrantes y otros actores sociales. Los proyectos y prácticas inspirados en el diálogo intercultural tienen el potencial de producir valores nuevos e inclusivos.

Palabras Clave: Políticas culturales, diversidad cultural, diálogo intercultural, desarrollo sostenible, ciudad. 


\begin{abstract}
Introduction: The globalized world has facilitated the proliferation of contacts between cultures. This situation has led to cultural and social transformations, often resulting in deep social divergences. Faced with this changing reality, a new debate challenges public policy. And the power of culture as a requirement for peace, a source of wellbeing and as a resource for sustainable development is more important than ever.

In order to know the potential of cultural policies in the territory based on a cultural diversity and intercultural dialogue context, this research focuses on cultural policies developed in the municipality of Botkyrka, south of the province from Stockholm (Sweden), through the case study of the Mångkulturellt centrum foundation.

Method: The qualitative method of research is applied in the study, developing a descriptive and exploratory work, where theory and logic are linked. Through the interpretive paradigm we have managed to study a particular context and emphasize the understanding of human experience. The three techniques applied are the study of the case, the content analysis and focused interviews (semi-structured) to experts from the foundation.

Results: Mångkulturellt centrum case allows us to study the detailed analysis of the foundation as a project that improves cultural diversity and intercultural dialogue in the territory, we identify successful actions, and we detect challenges faced by local policies at the present.

Conclusion: The research corroborates the importance of having public organizations that achieve intercultural competences, which are open to intercultural dialogue and work together with the immigrants themselves and other social actors. Projects and practices inspired by intercultural dialogue have the potential to produce new and inclusive values.
\end{abstract}

Keywords: Cultural policies, cultural diversity, intercultural dialogue, sustainable development, city. 


\section{Introducción}

Como en otras parcelas de la realidad de nuestras sociedades contemporáneas, la cultura se ha visto alterada por diferentes factores que confluyen con más intensidad en la actualidad. En el ámbito de las políticas públicas entendemos que éstas tienen que evolucionar de acuerdo a la realidad social y cultural de cada momento. Y las políticas culturales responden a las características de un contexto en un periodo temporal determinado, estableciéndose un diálogo entre las necesidades, problemáticas y situaciones de una realidad y las políticas como respuesta a este panorama. De esta forma y dependiendo del grado de dinamismo de esta relación pueden valorarse la adecuación de sus respuestas o la idoneidad de las formas de organización para un momento concreto en el devenir histórico.

La diversidad cultural no es un fenómeno nuevo pues la historia está marcada por numerosos movimientos migratorios intercontinentales. Sin embargo, en las últimas décadas, la diversificación cultural ha adquirido cada vez más importancia a consecuencia de una serie de cambios sustanciales en nuestras vidas. La globalización ha comprimido el espacio y el tiempo a una escala sin precedentes y la alta movilidad de personas, que a menudo se produce sin aculturación a los marcos culturales existentes, crea nuevas y radicales yuxtaposiciones culturales, encuentros, intercambios y mezclas. Estos desarrollos transculturales son ahora una preocupación importante para los mecanismos nacionales establecidos (Robins, 2006). El profesor Tony Bennett (2001), ya señalaba a inicios del siglo XXI como la diversidad cultural, en todas sus formas, plantea un profundo desafío a las formulaciones tradicionales de la política cultural y a nuestra comprensión de los intereses públicos a los que sirve esta política. En la mayoría de los países, el paisaje artístico y cultural no ha evolucionado para reflejar las realidades de un paisaje social cambiado. Esta brecha amenaza con socavar la legitimidad de las instituciones culturales y la política pública que las apoya. El cambio de la homogeneidad a la diversidad como nueva norma social requiere un replanteamiento de los procesos, mecanismos y relaciones necesarios para el desarrollo de la política democrática en sociedades diversas.

Desde el punto de vista del derecho de la cultura y las relaciones culturales internacionales, la UNESCO dio el primer paso en el año 2001 con la aprobación de la Declaración sobre la diversidad cultural (UNESCO, 2018a), cuyo texto anunciaba la necesidad de un tratado sobre este asunto. Así, la Convención sobre la protección y 
promoción de la diversidad de las expresiones culturales llegó en el año 2005 (UNESCO, 2018b), con su entrada en vigor en el año $2007^{1}$. A este gran avance legal y de notorias repercusiones, se unen numerosas iniciativas promovidas por la UNESCO cuyo resultado da testimonio del rico debate que la organización dirige sobre los desafíos de la diversidad cultural hasta el día de hoy (Barreiro, 2011; Prieto y Velasco, 2016); entre las conferencias, las mesas redondas, coloquios, seminarios y otras reuniones, así como informes, publicaciones y estudios destacamos dos trabajos (UNESCO, 2018c): Mapping cultural diversity. Good practices from around the globe (UNESCO, 2010) y Unesco Word Report. Investing in Cultural Diversity and Intercultural Dialogue (UNESCO, 2009).

En este contexto y desafiante panorama, donde aún las polémicas son vigentes, unos de los objetivos principales a los que se enfrenta el replanteamiento del diseño de las nuevas políticas culturales es la incorporación de la cultura en las políticas para el desarrollo. Entre los objetivos principales que pudieran acoger estas nuevas políticas podríamos señalar las siguientes, si bien estos objetivos se modulan o priman unos sobre otros dependiendo el país y las características de la sociedad del territorio específico (UNESCO, 2011): fomentar políticas innovadoras que promuevan las formas de diversidad cultural, facilitando el diálogo intercultural, la convivencia y el desarrollo sostenible; ampliar el especio democrático abarcando la diversidad; prestar apoyo al pensamiento creador y a la investigación innovadora para analizar los vínculos entre la cultura y la diversidad cultural, el desarrollo y la coexistencia armoniosa; prestar apoyo a las políticas locales, regionales y mundiales en las que se establezca un vínculo entre consideraciones de índole cultural, social, económica y medioambiental (políticas transversales) y mejorar la cooperación internacional para las políticas culturales con la

\footnotetext{
${ }^{1}$ En el artículo 4 de la Convención se recogen las siguientes definiciones: "1. Diversidad Cultural. La "diversidad cultural" se refiere a la multiplicidad de formas en que se expresan las culturas de los grupos y sociedades. Estas expresiones se transmiten dentro y entre los grupos y las sociedades. La diversidad se manifiesta no sólo en las diversas formas en que se expresan, enriquecen y transmiten el patrimonio cultural de la humanidad mediante la variedad de expresiones culturales, sino también a través de distintos modos de creación artística, producción, difusión, distribución y disfrute de las expresiones culturales, cualesquiera que sean los medios y tecnologías utilizados (...) 8. Interculturalidad. La "interculturalidad" se refiere a la presencia e interacción equitativa de diversas culturas y la posibilidad de generar expresiones culturales compartidas, adquiridas por medio del diálogo y de una actitud de respeto mutuo." Además de estos términos expuestos quisiéramos recoger el significado del término multiculturalismo, que será empleado en varias ocasiones a lo largo del artículo y así apreciar la diferencia con la interculturalidad. Multiculturalismo se entiende como la convivencia de personas identificadas con culturas de naturaleza variada en un mismo espacio, respetando las identidades culturales en una mutua convivencia representada en el espacio público y político (Usallán, 2012).
} 
elaboración de datos e indicadores o con formación a profesionales en el ámbito de la cultura, entre otras medidas

De esta forma, la cultura debe ser revisada no sólo en su ámbito per se sino, también, y dada su rica diversidad, como fuente, baza o inspiración para el desarrollo ${ }^{2}$. El desarrollo sostenible se experimenta en todos los niveles, pero especialmente a nivel local y requiere de espacios y procesos para la participación ciudadana, el debate y la toma de decisiones. Son los gobiernos locales quienes pueden activar estos aspectos para que los ciudadanos ejerzan sus derechos, amplíen sus capacidades, protagonicen el presente y decidan el futuro. La incorporación de la dimensión de la cultura es un elemento necesario para desarrollar estas líneas conjuntamente con los ámbitos de lo social, ambiental y económico, según defiende la Agenda 21 de la Cultura (Ciudades y Gobiernos Locales Unidos - CGLU, 2015). La integración de la cultura en las políticas de desarrollo permite: contribuir a la protección y la promoción de la diversidad de expresiones culturales; favorecer el acceso y la participación de todos, en particular de los grupos desfavorecidos, en la creación y la producción de expresiones culturales, y disfrutar de ellas y, mantener la cohesión social, combatir la violencia por medio de actividades culturales que valoricen los derechos humanos y la cultura de paz y refuercen entre los jóvenes el sentir de que pertenecen a su sociedad (UNESCO, 2012).

En este amplio y complejo contexto, los retos y desafíos que se plantean son numerosos. Los investigadores ${ }^{3}$ trabajan aportando nuevas ideas al mismo tiempo que los gobiernos locales comparten experiencias con ánimo a desarrollar acciones adecuadas en cada territorio ${ }^{4}$. Los temas tratados principalmente son la capacitación (fortalecer el desarrollo de capacidades en la planificación y diseño de proyectos culturales, con especiales competencias interculturales favoreciendo el apoyo al diálogo intercultural y la convivencia), el planteamiento con múltiples partes interesadas (crear vínculos explícitos entre organizadores del ámbito de la cultura y organizaciones que se ocupen de la diversidad cultural y diálogo intersectorial), la gobernanza innovadora para la cultura (establecer equipos de trabajo apropiados sobre la diversidad cultural),

\footnotetext{
${ }^{2}$ Para una mejor comprensión sobra la dimensión de la cultura en las acciones de desarrollo, documentos, encuentros internacionales, instrumentos normativos o documentos de referencia internacionales, se recomienda la consulta del enlace de Organización de Estados Iberoamericanos - OEI (2018). Cultura y Desarrollo. Obtenido de http://www.oei.es/historico/cultura/cultura desarrollo.htm

${ }^{3}$ Destacamos la relevancia de los estudios aportados por Ricard Zapata-Barrero (Universidad Pompeu Fabre), Jaquim Rius-Ulldemolins (Universidad de Valencia), Jordi Pascual i Ruiz (Universitat Oberta Catalunya), Nancy Duxbury (Universidade de Coimbra), Phil Wood (urbanista), Tony Bennett (Wester Sidney University) y Pierre-Michel Menger (Collègede France).

${ }^{4}$ Más información en Council of Europe (2018).
} 
remodelar las infraestructuras culturales (adaptar a nuevos contenidos y objetivos de las políticas culturales), nuevos tipos de asociación (fomentar colaboraciones trasnacionales), legislación (adaptar las legislaciones nacionales a los nuevos instrumentos que propician la UNESCO en estrategias operacionales y eficaces) y, creación y difusión de conocimientos sobre los nuevos ámbitos de las políticas (seleccionar y utilizar instrumentos de referencia, cartografías, buenas prácticas, estudios claves y otros) (UNESCO, 2011).

Uno de los autores actuales de referencia en relación al entendimiento de la cultura como transformador de la sociedad y como enfoque teórico alternativo a la instrumentalización de la cultura, es el australiano Jon Hawkes. Su trabajo titulado The fourth pillar of sustainability. Culture's essential role in public planning es decisivo para el entendimiento de la necesidad de estructurar el nuevo pilar para la sostenibilidad y el desarrollo local. Para Hawkes (2001), las acciones para el desarrollo de las sociedades descansan sobre cuatro pilares: el pilar económico, el pilar social, el pilar ecológico y, por último, el cuarto pilar que es la cultura. El objetivo es crear las bases conceptuales para que la cultura se convierta en el eje de nuestras políticas locales, aceptando que la vitalidad cultural es esencial para una sociedad sólida y saludable del mismo modo que lo son la igualdad social, la responsabilidad medioambiental y la viabilidad económica, y dado que la cultura reside en la gesta humana, entonces, necesitamos un camino para asegurar que toda la actividad pública se evalúe desde una perspectiva cultural (Pascual i Ruiz, 2007).

Desde este enfoque, el cambio de rol social de la cultura no debe sólo incluir una transformación de los objetivos sino también la forma de concebir la relación entre cultura y sociedad, es decir, pasar del paradigma de desarrollo implementado desde las élites (top-down) a un paradigma de la sostenibilidad que se base en la participación de la comunidad y el desarrollo social integral (Duxbury, 2008). Actualmente esta línea de pensamiento se plantea como alternativa a la "insostenibilidad" de la ciudad creativa, sustituyendo los debates anteriores sobre democratización cultural versus democracia cultural, consolidándose como uno de los futuros ejes de definición de la relación entre sociedad y cultura. No obstante, tampoco puede decirse que sea un paradigma coherente en sí mismo sino abierto a una viva discusión sobre sus implicaciones. En este sentido, la cultura facilitaría la transmisión de pautas de comportamientos individuales y colectivos más sostenibles, así como su traslación de este reto en el ámbito de la agenda política y la planificación económica y urbanística. Con esta perspectiva, la cultura 
puede aportar un equilibrio en la relación humanidad-economía-naturaleza, favorecer una actitud abierta al multiculturalismo y a un localismo tolerante, preservando las entidades locales, sus modos de producción y su urbanismo. Así, la cultura es vista como una herencia, un capital a promover contra las corrientes homogeneizadoras y como un vehículo para transmitir los valores de una sociedad sostenible ${ }^{5}$.

En virtud de ello, algunos países han implementado los primeros pasos en esta dirección y así podemos hablar del ejemplo de Nueva Zelanda donde a través de la Ley de Gobierno Local de 2002, el Ministerio de Cultura y Patrimonio del país promovió un modelo de bienestar comunitario con cuatro dimensiones interconectadas e interdependientes (culturales, ambientales, sociales y económicas) y desarrolló una serie de indicadores para guiar los Consejos de la ciudad y en cada área ${ }^{6}$. O la nueva visión y enfoque aportados por el Comité Asesor Externo de Ciudades y Comunidades del gobierno canadiense en el año 2006 con base en un modelo de desarrollo sostenible de cuatro pilares el cual sirvió para la creación de planes de sostenibilidad a largo plazo en las comunidades ${ }^{7}$.

Basándonos en el texto fundamentado por Jon Hawkes y siendo necesario desarrollar un nuevo perfil de la política cultural que contribuya a que la cultura se convierta en uno de los pilares de la sostenibilidad, la UNESCO recomienda la operacionalización de la relación entre sostenibilidad, cultura y sociedad teniendo en cuenta las siguientes recomendaciones (Ciudades y Gobiernos Locales Unidos-CGLU, 2009):

- Una planificación cultural integrada en la planificación nacional y acompañada y facilitada por una infraestructura adecuada.

- Procesos institucionales que promuevan los vínculos entre la cultura y la cohesión social, la sostenibilidad y el bienestar de la población. Creación de sinergias entre distintas políticas públicas.

- Mantenimiento y renovación de los procesos conscientes de las relaciones interculturales e interreligiosas a favor de un desarrollo comunitario positivo.

\footnotetext{
${ }^{5}$ Para más información, se recomienda la consulta del texto redactado por Duxbury et al. (2012).

${ }^{6}$ Más información en la página web del Ministerio de Cultura y Patrimonio (Ministry for Culture and Heritage) (2018): http://mch.govt.nz/what-we-do/our-projects/completed/cultural-well-being

7 Más detalles en Integrated Community Sustainability Planning - A Background Paper (2005): https://www.creativecity.ca/database/files/library/final icsp_discussion_paper.pdf
} 
- Identificar los caminos para hacer efectivo el reconocimiento, salvaguardia y utilización de los diversos sistemas de conocimiento para un desarrollo sostenible.

- Establecimiento de estrategias para promover las competencias interculturales en la planificación y un nuevo consenso cívico en las administraciones públicas y en las organizaciones de la sociedad civil.

- Expansión e innovación en la actividad del sector cultural tomando en consideración las ideas y las prácticas más recientes con las cuestiones interculturales, la cultura popular, promoción de la diversidad de expresiones culturales, la salvaguarda de los bienes tangibles e intangibles, etc.

- Mecanismos de coordinación efectiva en el nivel nacional y local para facilitar la implementación de aproximaciones innovadoras traspasando fronteras institucionales o geográficas.

El primer paso para abordar la diversidad cultural y el diálogo intercultural en la sociedad es reconocer la diferencia, defender la diversidad y promover las libertades culturales. Y ello, sólo es posible en la medida en que seamos conscientes de los conflictos que surgen en las sociedades multiculturales por el reconocimiento de la diversidad. La experiencia ha demostrado que cuando se intenta crear un tejido nacional negando las diferencias existentes se producen reacciones culturales violentas y la única vía para resolverlas y convivir con ellas es abordarlas (Programa de las Naciones Unidas para el Desarrollo - PNUD, 2004).

Suecia, históricamente, representa uno de los ejemplos más destacados de una política multicultural oficialmente declarada tras la Segunda Guerra Mundial. La primera política cultural del país fue establecida por el gobierno en 1974. Aunque el proyecto final de ley adoptó disposiciones para la promoción de la diversidad cultural, la participación de los inmigrantes en la vida cultural y la preservación de sus propias culturas; la idea de financiar un programa activo fue rechazado a la espera de los resultados obtenidos de una comisión de investigación. Fue a partir de este momento cuando las políticas multiculturales suecas comenzaron a tomar forma y han ido cambiando y transformándose acordes a la diversidad cultural de la sociedad del país y de los debates políticos planteados. Ya en la década de los noventa, el gobierno del partido Socialdemócrata creó una comisión de investigación independiente con el objetico de analizar los desafíos del futuro. En el texto final, denominado Dirección de 
Políticas Cultural (Kulturpolitikens Inriktning), reconocía que no se habían logrado las disposiciones sobre los inmigrantes y minorías nacionales en la política cultural de 1974, y consecuentemente, en 1996 se adoptó la segunda política cultural nacional con el objetivo claro de aumentar la participación de las minorías en la vida cultural, alentar la diversidad cultural y los intercambios culturales a nivel internacional provocando encuentros entre diferentes culturas. Su propósito estaba encaminado especialmente a combatir la xenofobia y el racismo, estimulando la integración. Dos años después y a través de un nuevo dossier redactado por la comisión Forum por la Cultura Mundial (Forum för Världskultur) recomendó, entre otros aspectos, la designación de consultores multiculturales en las regiones del país y otorgar la responsabilidad de promover el multiculturalismo al Consejo Nacional de Asuntos Culturales de Suecia. A comienzos del siglo XXI, la Ministra de Cultura Marita Ulvskog (partido Socialdemócrata) creó una agenda para los años 2003-2006 de cuyos objetivos destacaron la instauración del año dedicado al multiculturalismo (2003), el aumento del conocimiento de la población de artistas nacidos en el extranjero, el apoyo a publicar sus trabajos y una investigación sobre cómo las instituciones y otros actores de la sociedad implementan el multiculturalismo. Desafortunadamente los resultados demostraron que las instituciones culturales públicas habían avanzado poco con respecto a la implementación del multiculturalismo hasta la fecha ${ }^{8}$.

Ya durante los últimos cuatro años de gobierno sueco (2014-2018), las políticas específicas relacionadas con la diversidad cultural se han orientado en tres ejes: las políticas de diversidad e inclusión cultural, diálogo intercultural: actores, estrategias y programas y, la cohesión social y la política cultural. Para el gobierno de coalición del Partido Socialdemócrata y el Partido Verde gobernante en este período, la diversidad cultural y el trabajo contra el racismo han sido áreas prioritarias en la política cultural. Todos los presupuestos nacionales contemplaban medidas destinadas a apoyar la diversidad y la inclusión, incluido un mayor apoyo para las actividades de la sociedad civil con este enfoque. E igualmente una mayor prioridad a la diversidad cultural en las políticas dirigidas a las instituciones artísticas y del patrimonio (Museo Nacional de Historia y los Museos Nacionales de Cultural Mundial). Reconociendo a Suecia como

\footnotetext{
${ }^{8}$ Los estudios y publicaciones realizados por Karin Borevi (Södertons Högskolan) y Tawat Mahama (Mälmo Universitet) complementan una visión más detallada de la evolución de las políticas culturales en Suecia (Borevi, 2013; Tawat, 2014, 2017).
} 
una sociedad multicultural, el gobierno otorgó a las minorías nacionales ${ }^{9}$ y sus lenguas subvenciones para proyectos en los campos de la lengua, la literatura y publicaciones periódicas con contenido cultural. Por otro lado, el apoyo al diálogo intercultural fue promovido por todas las agencias gubernamentales, con la evaluación de las nueva Agencia Gubernamental para el Análisis Cultural. Entre los temas abordados en este campo se encuentran el problema del lenguaje pues debido a la inmigración, un gran porcentaje de la población habla otros idiomas además del sueco, las lenguas minoritarias nacionales reconocidas o las lenguas maternas (más de 150 idiomas se hablan en el país). Además, el Comité de Subvenciones de las Artes dirige un programa de estudio para artistas visuales (IASPIS), abierto a artistas de Suecia y del extranjero, y el Instituto Sueco tiene subvenciones para intercambio internacional en las disciplinas de las artes, la ciencia y los medios de comunicación. Por último y en relación a la cohesión social, el objetivo de la política cultural moderna es aumentar el acceso a la cultura para todos los que viven en Suecia, tanto a través del acceso a una cultura de alta calidad como al permitir que más personas practiquen actividades culturales y artísticas. Por lo tanto, la participación y la cohesión social pueden considerarse el núcleo de la política cultural sueca. La política de integración (a todos los niveles administrativos) está basada en la igualdad de derechos, responsabilidades y oportunidades para todos, independientemente de su origen étnico y cultural; cohesión social basada en la diversidad; desarrollo social caracterizado por el respeto mutuo, independientemente de los antecedentes y con un sentido de comunidad. La diversidad étnica y cultural es el punto de partida para configurar políticas generales en todos los sectores y en todos los niveles de la sociedad. Numerosos debates y documentos políticos actuales otorgan alta prioridad a abordar la segregación en la sociedad sueca, así como a los esfuerzos para combatir el racismo, la xenofobia y la discriminación étnica. Los esfuerzos de integración están centrados en la creación de oportunidades que permitan a las personas mantenerse económicamente y participar en la sociedad ${ }^{10}$.

\footnotetext{
${ }^{9}$ Las minorías nacionales oficialmente reconocidas son el pueblo sami, los finlandeses suecos, los tornedalers, los romaníes y los judíos. Todas las minorías nacionales tienen sus instituciones culturales nacionales, por ejemplo, el Teatro Sami, el Museo Sami Aitte, el Teatro Tornedalen, el Centro Cultural Roma en Mälmo y el Museo Judío. En 1999, cinco lenguas minoritarias fueron declaradas oficiales en Suecia: Sami (todas las variedades), Finlandés, Meänkieli (históricamente conocida como tornedal finlandés), Romaní Chib (todas las variedades) y el Yiddish.

${ }^{10}$ Información recogida de la plataforma Compendium of Cultural Policies and Trends, sistema de información y monitoreo de políticas culturales nacionales elaborada y actualizada por expertos que están en continuo contacto con los ministerios de los respectivos países. Más información en http://www.culturalpolicies.net/web/index.php
} 
Atendiendo a lo descrito, la presente investigación se pregunta ¿cuál es la potencialidad de las políticas culturales en el territorio como base de un contexto asentado en la diversidad cultural y el diálogo intercultural? Y para responder a esta pregunta el estudio se ha centrado en las políticas culturales elaboradas en el municipio de Botkyrka, Suecia, a través del estudio de la fundación Mångkulturellt centrum. El país escandinavo se caracteriza por ser uno de los estados de Europa donde conviven numerosos inmigrantes procedentes de muy diversos lugares en conflicto, y el municipio de Botkyrka es un territorio donde la cifra es muy elevada. De este modo y con ánimo a una mejor integración de estas personas, la administración local creó esta institución. Un centro creado en el año 1987, el más antiguo de Europa, con las finalidades de la investigación, la educación y la cultura. Teniendo como ambiciones y objetivos estudiar y promover una sociedad donde la diversidad y la migración formen parte de la cultura sueca.

Existen varias razones por las que esta investigación resulta pertinente y plenamente justificada. Por un lado y en relación al objeto de estudio, la incorporación de las políticas culturales en el debate sobre la gestión de la diversidad es fundamental en la sociedad globalizada que vivimos. Es importante mostrar la dimensión socializadora y de fomento de la ciudadanía que supone la cultura, especialmente en contextos urbanos socioeconómicos más desfavorecidos. Por ello, este trabajo permitirá comprender y ampliar el espectro de análisis, aportando puntos de reflexión y complementando los actuales estudios. Por otro lado e identificando las políticas culturales como un conjunto de acciones, el estudio puede aportar una visión importante como ejemplo de buena práctica. Ofreciendo una respuesta a estrategias nuevas y creativas de solución a problemas comunes, identificando rasgos que puedan ser aplicables a otros contextos y representando un modelo que pudiera ser susceptible de ser imitado. Por último y en conexión con la implementación, el estudio de la fundación Mångkulturellt Centrum permitirá esclarecer las dificultades a las que se enfrentan las políticas culturales basadas en la diversidad cultural y el diálogo intercultural. Siendo un estudio pertinente en tanto que puede ser útil para descubrir cuáles son los puntos más sensibles a la hora de idear y planificar estrategias futuras.

\section{Método}

El método cualitativo de la investigación es el aplicado en el estudio, desarrollando un trabajo descriptivo y exploratorio, donde la teoría y la lógica están 
unidas. Tres son las etapas desarrolladas en esta investigación: formulación, diseño y ejecución y cierre. El proceso de formulación ha consistido en la fase exploratoria (acercamiento a la realidad, revisión de la documentación existente, observación o entrevistas informarles), la fase de mapeo (situarse en el terreno conociendo los actores, participantes o acciones), la fase de muestreo (selección de actores o eventos que serán abordados), la exploración de la literatura existente relacionada con la temática tratada y depurándola en categorías y, la formulación de la pregunta de investigación. Dentro de la etapa de diseño y ejecución se ha procedido a la delimitación en profundidad y extensión del proceso de recolección de datos, encauzar el inicio de la recolección y su delimitación, la implementación y la ejecución de la investigación y, por último, el análisis de la información. La etapa de cierre ha tratado la organización de los datos, la fase descriptiva de la organización de los datos, la fase de segmentación de los datos a partir de categorías, la fase de reagrupación de los datos para conformar categorías de segundo orden, finalización de recogida de información, preparación de los datos, análisis de la información y fase de elaboración del informe.

A través del paradigma interpretativo hemos conseguido realizar el estudio de un contexto particular y enfatizar la comprensión de la experiencia humana. La metodología interpretativa hace hincapié en la fidelidad al asunto investigado, defiende la complementariedad de las perspectivas aportadas por el investigador y por los participantes, dando como resultado un concepto de verdad entendido como el grado de correspondencia establecido entre el relato del investigador sobre la experiencia de los participantes y la visión de éstos.

Las tres técnicas aplicadas son el estudio del caso (acercamiento a un tema de interés de mayor amplitud focalizándolo en un ejemplo concreto, Mångkulturellt centrum), el análisis de contenido (análisis de la realidad social escogida mediante el estudio de documentos) y entrevistas focalizadas (semiestructuradas) a expertos y responsables del propio centro de estudio del caso. En cuanto a esta última, fue elaborado un guión con los siguientes apartados: introducción, perfil de la persona entrevistada (género, nombre y apellido, edad, desempeño laboral, años de experiencia, nivel de estudios e idiomas que habla) y preguntas formuladas bajo el epígrafe "Estrategias interculturales en la fundación Mångkulturellt centrum”. Las entrevistas buscaron la reflexión sobre el discurso basado en la autoevaluación crítica de los actores implicados en el proyecto Mångkulturellt centrum. Las cuatro preguntas formuladas fueron organizadas en cuatro dimensiones que sirvieron para proponer un marco 
interpretativo y para reflexionar sobre las estrategias empleadas para fomentar el vínculo entre interculturalidad y política cultural:

Conceptual. ¿Cómo entiende la interculturalidad en el marco de su trabajo?

Objetivos. ¿Cuáles son los principales objetivos buscados en su trabajo?

Límites. ¿Cuáles son los principales problemas encontrados?

Mejoras. ¿Cuáles son los retos para mejorar el vínculo entre la diversidad, la interculturalidad y la política cultural en el marco de su trabajo?

\section{Resultados}

En este apartado queremos reflejar tres aspectos fundamentales partiendo de que el estudio de caso de Mångkulturellt centrum permite estudiar cuál es la potencialidad de las políticas culturales en el territorio teniendo como base un contexto asentado en la diversidad cultural y el diálogo intercultural. El primer aspecto se relaciona con el análisis de la Fundación como proyecto que mejora la diversidad cultural y el diálogo intercultural en el territorio, y para ello se detallan sus rasgos concretos, creando de este modo una herramienta de reflexión y trabajo con una vertiente descriptiva. Seguidamente, identificamos acciones concretas que mejoran la diversidad cultural y el diálogo intercultural en el territorio, consiguiendo reconocerlas y detallar sus aspectos. Y, por último, detectamos desafíos y problemáticas a las que se enfrentan las políticas locales enfocadas al fomento de la diversidad cultural y el diálogo intercultural.

\subsection{Presentación y emplazamiento de Mångkulturellt Centrum}

Mångkulturellt centrum (MKC) es una fundación del municipio de Botkyrka, ubicada en la zona residencial de Fitja. Constituida en el año 1987, la base de su creación fue el compromiso cultural e histórico, con la convicción de que la inmigración contemporánea en Suecia es tan importante como la propia emigración histórica del país (Roth y Hertzberg, 2012). Su deseo es promover activamente un clima social favorable e incrementar el conocimiento sobre las nuevas condiciones de la sociedad sueca, apoyando a las instituciones suecas que se ocupan de la migración y la globalización a través de la investigación (multidisciplinar con énfasis en un enfoque etnográfico), de la educación y de las actividades culturales. Y de igual modo, desarrollar y difundir el conocimiento de los factores sociales y culturales que facilitan la creación de una sociedad sostenible. Las ambiciones del centro son estudiar y promover una sociedad 
donde la diversidad se refleja en la imagen nacional de Suecia y donde la migración es una parte natural del patrimonio cultural sueco.

La economía de la Fundación se basa en el apoyo económico recibido del propio municipio de Borkyrka, del Consejo del Condado de Estocolmo -Cultura y Educación (Stockholms läns landsting-Kultur och tbildningsnämnden) y del Consejo Sueco de las Artes (Statens Kulturådet) ${ }^{11}$, complementados con los ingresos conseguidos a través de servicios ofrecidos por el propio centro: educación e investigación, alquiler de espacios para conferencias, una tienda y las producciones de la editorial.

Por sus características y particularidades, Botkyrka puede interpretarse como el sismógrafo que detecta los cambios en la sociedad local, que posteriormente se desarrollan a un nivel nacional. La cuestión de la diversidad cultural, que en los años ochenta se centró en gran medida en Botkyrka y en algunas otras áreas metropolitanas, es hoy relevante en toda Suecia (Swedish Association of Local Authorities and Regions - SALAR, 2009).

Botkyrka es el municipio más diverso de Suecia, junto con los municipios de Södertalje (Condado de Estocolmo) y Haparanda (Condado de Norrbotten), y el primer municipio donde la población sueca ya no es la mayoría, el mayor segmento demográfico entre muchas minorías ${ }^{12}$. Entre el 2010 y 2012, la proporción de residentes con antecedentes extranjeros aumentó al $55 \%$ en total y el $65 \%$ entre todo los niños y jóvenes ( 0 a 18 años) del municipio, la población con antecedentes extranjeros incluía cerca de 160 países de orígenes diferentes. Ya en el año 2010, Botkyrka se convirtió en el municipio con la mayor proporción de residentes con antecedentes extranjeros, con un $53,2 \%$ de los cuales la mayoría tenían antecedentes no europeos ${ }^{13}$.

\footnotetext{
${ }^{11}$ Para más información sobre las instituciones mencionadas se recomienda la consulta de los siguientes enlaces: Stockholms läns landsting. (2018). Recuperado de http://kultur.sll.se/ y Statens Kulturrådet. Recuperado de http://www.kulturradet.se/

${ }^{12}$ Más información y detalle en el enlace de la Agencia de Estadística de Suecia (Statistika centralbyrån). Nuevos ciudadanos suecos de más de 160 países (Nya svenska medborgare från drygt 160 länder), 2103-2018:https://www.scb.se/hitta-statistik/statistik-efter-amne/befolkning/befolkningens-

sammansattning/befolkningsstatistik/pong/statistiknyhet/folkmangd-och-befolkningsforandringar-20172/. Si bien en la nota 9 señalábamos las cinco minorías nacionales suecas y sus lenguas a las que hacen alusión las políticas nacionales en Suecia, en este caso se hace mención a una definición de minorías con un espectro más amplio y admitido igualmente por el derecho internacional (UNESCO). Es decir, las minorías de inmigrantes, refugiados, étnicas, culturales y, lingüísticas y religiosas.

${ }^{13}$ Es importante señalar que el término "inmigrante" fue descartado oficialmente en 1999 y sustituido por el término "antecedente extranjero" (utländsk bakgrund). Personas de "origen extranjero" es, por tanto, el término oficial y la categoría estadística en Suecia que describe a los migrantes y a sus hijos. La categoría demográfica de origen extranjero incluye a todos los residentes registrados en Suecia, independientemente de su nacionalidad, que hayan nacido en el extranjero o hayan nacido con padres nacidos en el extranjero. Más información en Kulturdepartament (1999). Begreppet invandrare. Användningen i lagar och förordningar. Estocolmo, Suecia: Fritzes Offentliga Publikationer.
} 
Botkyrka comienza a recibir inmigración en los años cincuenta y sesenta cuando la afluencia de trabajadores a la industria sueca consistía en inmigrantes nórdicos y europeos, principalmente de países como Finlandia, Polonia, Yugoslavia, Grecia, España y Alemania. A finales de la década de 1960 y principios de la década de 1970 llegaron los turcos y kurdos, así como minorías cristianas de Turquía, Siria y Líbano. A partir de la década de 1970, otros grupos migratorios no europeos, no protestantes, no blancos y no occidentales siguieron una época en que la inmigración laboral fue reemplazada por la inmigración de refugiados. Además de la inmigración de Turquía, Siria, Líbano e Irak, otras poblaciones minoritarias notables en Botkyrka han sido los chilenos, colombianos, pakistaníes, bangladesís, filipinos, chinos, afganos, marroquíes, indios, iraníes y etíopes, así como polacos, bosnios y rusos. A partir de los años ochenta y particularmente de los noventa, la inmigración no occidental sustituyó a la inmigración europea, convirtiendo a Botkyrka septentrional en la zona fuertemente dominada por residentes procedentes de países no occidentales (Botkyrka Kommun, 2014). Actualmente el municipio de Botkyrka tiene uno de los porcentajes más latos de inmigrantes de primera y segunda generación en Suecia y, sus rasgos como comunidad multicultural lo atestiguan su gran iglesia ortodoxa siríaca en Hallunda y la mezquita ubicada en Fitja, ambas zonas urbanas ubicadas al norte del Botkyrka. Si en el año 2016, el 56,4\% de la población tenía al menos un padre nacido en otro país, los datos del año 2017 señalan que el 58,56\% de la población del municipio son personas de origen extranjero, es decir, personas nacidas fuera de Suecia o con padres nacidos fuera de Suecia.

Entre febrero del 2013 y mayo del 2014 un equipo de investigación de Mångkulturellt centrum elaboró un estudio, bajo el auspicio de la fundación Open Society Foundations, para abordar las preocupaciones de los grupos de inmigrantes en el municipio de Botkyrka y proporcionar así nuevos datos que fueran valorados en los debates públicos sobre integración, cohesión e inclusión social ${ }^{14}$. Los resultados obtenidos mostraban a Botkyrka como el primer municipio de Suecia de mayoría

\footnotetext{
${ }^{14} \mathrm{El}$ informe se basa en 12 reuniones de grupos focales con 70 habitantes del sur de Botkryka y en 22 entrevistas individuales con personas que trabajan y participan en la misma área. En el reclutamiento de los participantes del grupo de enfoque, se utilizó el término "mayoría de suecos". Este denota a aquellos participantes que se auto categorizan o identifican como pertenecientes a la mayoría de la población sueca, independientemente de si son de origen sueco o inmigrantes o descendientes de inmigrantes de otros países occidentales (categoría abierta y negociable). Si bien el informe aborda los puntos de vista y las experiencias de la mayoría de suecos en el sur de Botkyrka, los datos son potencialmente aplicables a la situación de la mayoría de suecos en el Gran Estocolmo en general porque el patrón de segregación y las estadísticas en el municipio de Botkyrka son similares a toda la región capital.
} 
minoritaria, donde la mayoría sueca constituye el grupo demográfico más grande pero no la mayoría de la población. Pocos suecos mayoritarios están marginados y no parecen ver a los inmigrantes como amenaza para su bienestar económico. Sin embargo, no los ven ni a ellos ni a sus hijos como suecos y consideran que la inmigración es hostil a los valores y al estilo de vida suecos. Tales actitudes son un factor importante en su continua marginación que, a su vez, amenaza la cohesión, la prosperidad social y evita que los inmigrantes desarrollen su potencial contribuyendo a la vida económica, cívica y cultural del país (León, 2011). Por ello es necesaria una renovación de la identidad sueca que se base en el profundo compromiso de Suecia con los derechos humanos y la igualdad, y que garantice que todos puedan participar y contribuir a la sociedad del país, sintiéndose cómodos con la nueva realidad de una población diversa. El debate abierto en diferentes niveles podría promover este proceso con pasos prácticos hacia una identidad más renovada a través de la apertura de la cultura pública en Suecia y mostrando su diversidad en mayor grado en teatros, museos o en los espacios públicos. O generando medidas para combatir la discriminación y los delitos de odio con iniciativas de sensibilización a los empleadores, incluyendo la diversidad en el currículo escolar o la participación cívica y las actividades comunitarias más inclusivas (Daun et al., 1982; Hosseini-Kaladjahi, 2002; Open Society Foundation, 2014).

\subsection{Visión, misión y objetivos}

MKC trabaja por una sociedad donde la diversidad se refleja en la autoimagen nacional de Suecia y donde el fenómeno migratorio está incluido como parte integrante del patrimonio cultural sueco. Una sociedad en la que nadie tiene que sacrificar su propia cultura e identidad con el fin de lograr una participación e integración. Su misión es ser un foro y lugar de encuentro de diferentes formas de expresión, investigación, conocimientos y experiencias sobre la migración y la diversidad social y cultural. Las actividades desarrolladas conducen a mejoras sociales de forma activa, a un mayor conocimiento y a un aumento de las habilidades de las personas y las organizaciones, facilitando una sociedad sostenible ${ }^{15}$ (Mångkulturellt centrum - MKC, 2006).

\footnotetext{
${ }^{15}$ En el año 2017, Mångkulturellt centrum cumplió 30 años y a lo largo de esta trayectoria ha sido capaz de crear numerosas actividades y proyectos afines a los objetivos del centro. Además de los dos proyectos destacados y detallados unas páginas más adelante, First Generation_Esther Shalev-Grez y Att minas migrationen _ Nordiska Museet, quisiéramos hacer mención a dos proyectos de mediación cultural de gran transcendencia en la actualidad: $N o B o$ es un proyecto que trabaja con jóvenes y tiene como objetivo aprovechar la creatividad de los individuos con una gran comprensión de su realidad; Havat (que en turco y en árabe significa vida) es un proyecto para recién llegados y solicitantes de asilo cuyo propósito es
} 
Las memorias anuales de MKC nos reflejan cuatro objetivos en los que se afianza la labor del centro desde su creación en el año 1987 (MKC, 2006, 2008-2014, 2016 y 2017):

1. Producir conocimiento sobre la migración, la discriminación cultural, racial y condiciones de vida urbana.

2. Comunicar conocimientos en diálogo con el mundo académico, la sociedad civil, las empresas y el sector público.

3. Ser un actor activo en el municipio de Botkyrka (ámbito local), en el Consejo del Condado (ámbito regional) y en el gobierno estatal (ámbito nacional) con el objetivo de desarrollar adecuadamente la misión de MKC.

4. Crear y gestionar contactos internacionales que refuercen el conocimiento y la experiencia local, regional y nacional del trabajo desarrollado en MKC.

\subsection{Lineas de contenidos, acciones generales y acciones especificas destacadas}

Las numerosas acciones anuales desarrolladas por $\mathrm{MKC}$ en relación a sus objetivos podríamos enmarcarlas dentro de las siguientes líneas de contenidos (MKC, 2006, 2008-2014, 2016 у 2017):

- Aprender, entender y transformar (Lär, första och förandra)

MKC capacita y educa a grupos del sector público y privado, tales como organizaciones de la sociedad civil, empresas, autoridades gubernamentales y escuelas, tanto en las instalaciones del cliente como en los propios espacios de MKC. Los temas tratados son especialmente el racismo y como combatirlo en nuestras vidas cotidianas. Ayudan a las organizaciones y a las empresas a trabajar por la inclusión y la diversidad, creando sociedades y encuentros interculturales con espacio para todas las personas.

- Investigar y profundizar (Forska och fördjupa)

crear actividades culturales orientadas a este grupo objetivo, inspirándoles y dándoles más oportunidades para llenar su vida diaria con actividades creativas. Por otro lado, destacar también dos de las investigaciones vigentes del centro: Metodologiska laboratorier es un proyecto de investigación, en colaboración con la Universidad de Uppsala y Universidad de Sördertörn, que tiene como objetivo desarrollar métodos éticos y científicamente sustentables para producir conocimiento empírico sobre la discriminación por motivos de origen étnico, color de piel y religión; Förorten och folkbilningdrenässans, en colaboración con la Universidad de Gotemburgo, centra los análisis y estudios en los procesos de aprendizaje que están vinculados a la movilización de personas en áreas urbanas marginadas. En la última memoria anual publicada del centro (MKC, 2018), la fundación informa que durante el año 2017 recibieron a 28.720 visitantes de los cuales 3.590 eran niños y 1.300 jóvenes. Estas buenas cifras se complementan con la relevancia que el centro ha tomado a lo largo de todos estos años en el terreno académico, municipal y en el ámbito cultural. 
MKC es un centro enfocado al conocimiento. El enlace entre la práctica y la investigación ha sido siempre el corazón de su trabajo. MKC presenta un ambiente de investigación interdisciplinario que trasciende varios campos y problemas. Su foco de atención está ligado a tres áreas especiales: estudios críticos del patrimonio cultural, condiciones de vida urbana, así como raza, discriminación y carácter sueco o condición sueca (Svenskhet /Swedishness).

- Leer nuestras publicaciones (Läs våra publikationer)

MKC publica investigaciones y estudios realizados en el centro, pero también otras obras que se relacionan con los temas abordados en la organización. Algunas de estas publicaciones son empleadas con fines educativos.

- Colaboraciones (Samarbeta)

Para que MKC sea una plataforma y punto de referencia para reuniones e intercambios, es importante fomentar las colaboraciones. Los investigadores de MKC invitan y participan en discusiones locales, nacionales e internacionales con representantes del gobierno estatal, regional y local, sociedad civil y ámbito empresarial/privado.

- Experiencia y exploración (Upplev och Utforska)

Para difundir el conocimiento a un amplio público, produce exposiciones relacionadas con la visión de $\mathrm{MKC}$ desde perspectivas locales, nacionales y globales. MKC cuenta con dos áreas de exposición, una sala grande que acoge producciones propias $\mathrm{y}$, una sala pequeña donde el centro coopera con socios externos.

- Espacio creativo (Skapa kreativt)

Entendiendo el arte como una herramienta fundamental, el equipo de MKC trabaja especialmente con los niños pero también con adultos, con el fin de reunirlos alrededor de temas de pequeña o gran envergadura. En estos espacios se mezclan los pensamientos, las emociones, los colores y distintos materiales para crear un entorno variado en sus distintas facetas.

- Leer y reflexionar (Läs och Fundera)

La biblioteca de MKC cuenta con una de las colecciones más completas de libros, informes y artículos sobre migración, diversidad, origen étnico, discriminación, vida en el exilio y segregación. Los fondos son herencia de las bibliotecas en la Oficina Nacional de Inmigración (Statens invandrarverks 
biblioteket) y de la Agencia de Integración (Integrationsverkets biblioteket), y continúan incrementándose con nuevas publicaciones o textos resultantes de los debates, encuentros de investigadores o grupos de estudio actuales.

- Comer y beber ( $\ddot{t}$ och drick)

El restaurante "Tavernan" sirve comida vegetariana preparada en la propia cocina de $\mathrm{MKC}$, hecha con ingredientes frescos de temporada y con inspiración en todos los rincones del mundo. Un punto de referencia para nuevos visitantes y conocer otras culturas gastronómicas.

- Compras sostenibles (Shoppa hållbart)

La tienda de MKC reúne productos de comercio justo de todos los rincones del mundo y también locales, además de los libros publicados por la editorial MKC y otras relacionadas.

- Reunirse con nosotros y con otros en nuestros locales (Möt oss, varandra och andra $i$ våra lokaler)

MKC ofrece espacios donde reunirse en el corazón de Botkyrka, modernas instalaciones con diferentes dimensiones.

La finalidad más importante de la investigación elaborada por $\mathrm{MKC}$ es que los resultados puedan ser utilizados en el trabajo práctico cotidiano pues, es la aplicación práctica de los resultados de la investigación lo que permite mejorar la vida de los grupos objetivos potenciales (Mångkulturellt centrum - MKC, 2006). En este sentido, reflejamos a continuación dos ejemplos de acciones significativas de MKC.

\section{First Generation_Esther Shalev-Gerz}

Aunque MKC es una organización dedicada en gran medida al conocimiento, de manera regular desarrolla actividades culturales para lograr sus propósitos. El programa de exposiciones, iniciado en el año 2003, centra la atención en amplios temas culturales, artísticos y contemporáneos. Y su propósito general es discutir las diferencias dentro del alcance de la identidad humana, teniendo como objetivo establecer una perspectiva con base en la creación de igualdad y de calidad de vida.

En el año 2002, MKC inició la cooperación con la artista nacida en Lituania, Esther Shalev-Gerz. Con el proyecto First Generation, la artista convocó a personas procedentes de otros países, así como suecos de origen que vivían en Botkyrka en ese momento. Los voluntarios fueron invitados a participar en una reflexión sobre su identidad dentro de las múltiples referencias culturales existentes en el territorio. Un 
total de 34 personas acordaron colaborar en el proyecto cultural y fueron filmadas mientras respondían a una serie de preguntas relacionadas sobre su llegada al municipio de Botkyrka. El trabajo contribuyó de manera ejemplar a la misión de MKC, difundiendo la conciencia que facilita la comprensión y el discernimiento, lo que a su vez puede ayudarnos a entender los marcos de referencia de otras personas. La instalación de vídeo resultante destaca cómo el mundo está entrelazado cada vez más a través de la migración y la forma en la que la gente de todos los ámbitos étnicos se ha convertido en parte de un nuevo patrón social histórico ${ }^{16}$. La instalación tuvo una gran acogida entre el público sueco a lo largo del año 2004 y, durante el Año de la Diversidad Cultural 2006, realizó una gran gira internacional por diversos países: Kazán (Rusia), Dublín (Irlanda), París (Francia) y Bruselas (Bélgica) (Magnusson, 2006).

Att minas migrationen_Nordiska Museet

El Museo Nórdico (Nordiska Museet) y MKC crearon un acuerdo de colaboración en el año 2008 para desarrollar un proyecto donde quedaran recogidas las experiencias y vivencias de las personas que vienen a vivir a Suecia, bajo el nombre "Recordando la migración" (Att minas migrationen) ${ }^{17}$. El proyecto, que sigue vigente en la actualidad y no tiene límite de tiempo, está basado en la idea de saber más sobre la inmigración y la emigración con las propias experiencias de las personas. Y entendiendo que los movimientos de la población y la migración son una parte importante de la sociedad y la cultura actual.

La convocatoria está dirigida por tanto a todos los que se han trasladado a Suecia. Los textos aportados son conservados en el archivo del Museo Nórdico y estarán disponibles para futuras investigaciones y/o exposiciones. Y de esta forma, las narraciones contribuirán a la formación de la herencia cultural en Suecia y servirán de información para las próximas generaciones (Mångkulturellt centrum - MKC, 2009). El archivo del museo cuenta con un gran banco de experiencias que han sido acumuladas desde hace décadas, las cuales tienen una gran demanda por parte de los científicos con ánimo de conocer y profundizar en la acción diaria de la gente común; existe un gran interés por las propias vivencias de las personas y los distintos pensamientos existentes en el mundo.

\footnotetext{
${ }^{16}$ El vídeo puede ser revisado en el siguiente enlace: Esther Shalev-Gerz. (2018). First Generation. Recuperado de http://www.shalev-gerz.net/?portfolio=first-generation-2

${ }_{17}$ Más información en el enlace: Nordiska Museet. (2018). Att minas migrationen. Recuperado de https://www.nordiskamuseet.se/samlingar/insamling/beratta-oss/att-minnas-migrationen
} 


\subsection{Colaboraciones internacionales y reconocimiento}

Durante años, el equipo de profesionales de MKC ha establecido un entorno práctico basado en la experiencia que facilita fuertes enlaces a una variedad de expertos, técnicos y organizaciones a nivel internacional. Los contactos han sido continuos y las colaboraciones han dado como resultado diversos proyectos conjuntos. En este sentido, quisiéramos mencionar algunas de las colaboraciones más relevantes, si bien los ejemplos son numerosos.

MKC apoya actualmente al municipio de Botkyrka en relación al trabajo desarrollado en el programa Ciudades Interculturales (Intercultural Cities) del Consejo Europeo, pero también colabora en la Coalición de Ciudades contra el Racismo (European Coalition of Cities Against Racism-ECCAR). A propósito de esta última organización, MKC implementó un proyecto entre los años 2010 y 2013 cuyo objetivo principal era la mejora del impacto de las estrategias y acciones locales contra el racismo y la xenofobia, así como garantizar su sostenibilidad en el tiempo. En el caso de Botkyrka, la estrategia abordada fueron los llamados "círculos de investigación intercultural". Esta estrategia se basó en reunir ciudadanos de distintos colectivos y barrios, creando grupos de diálogo, con la idea de que una interacción prolongada con los actores locales contribuye a una comprensión profunda y cualitativa de cómo se manifiestan el racismo y la discriminación desde la experiencia vivida por los distintos colectivos. Con la ayuda de un investigador, se analizaron las condiciones de vida de los ciudadanos para identificar procesos discriminatorios en el municipio y formular recomendaciones dirigidas a las autoridades sobre cómo erradicar dichos procesos (European Cities Against Racism - ECAR, 2013).

Por otro lado y a propósito de la participación de MKC en el programa Ciudades Interculturales, no podemos dejar de mencionar que la Fundación fue reconocida como buena práctica en el año 2017. Este programa emblemático del Consejo Europeo ayuda a las autoridades locales a diseñar políticas basadas en la aplicación de un nuevo modelo de gestión de la diversidad denominado "integración intercultural". El modelo de política se basa en la noción de "ventaja de la diversidad", es decir, tratar a los inmigrantes como un recurso para el desarrollo económico, social y cultural local, y no sólo como grupos vulnerables que necesitan apoyo y servicios. La integración intercultural implica una reorientación estratégica de la gobernanza urbana y de las políticas para fomentar una representación adecuada, una mezcla intercultural y una interacción positivas, y la capacidad institucional para garantizar un acceso equitativo a 
los derechos y oportunidades para todos (Council of Europe, 2018; Rojas, 2010). Pero, ¿cómo dimensionamos lo que es una buena práctica intercultural? Los adjetivos y características que definen una buena práctica intercultural son aquellos aspectos que referencian igualmente la política intercultural. De este modo, podríamos identificar tres aspectos mínimos que detectamos en una buena práctica intercultural y que claramente están recogidos en MKC (Pinyol, 2013):

1. Objetivos. Estar dirigido a una población diversa y no responder a una única forma de expresión de diversidad. Aunque no es sólo una cuestión de orígenes, sino que también afecta a características como la edad, el sexo, la formación, la lengua, la religión, etc. La buena práctica intercultural es aquella que asume la sociedad diversa como un todo, y es a este todo al que intenta proporcionar un mejor servicio.

2. Procedimiento y recursos. Fomentar la interacción entre las diferentes formas de expresión de la diversidad. La buena práctica intercultural no es un servicio que la Administración proporciona al ciudadano sin más, sino que es un instrumento que permite igualmente una mayor interacción con el resto de sus conciudadanos. Tiene un claro elemento de innovación respecto a modelos de funcionamiento más tradicionales.

3. Resultados. Garantizar la socialización en la diversidad y el conocimiento intercultural. La buena práctica intercultural es un mecanismo de creación y distribución del conocimiento, y en la medida que son efectivas proporcionan validez a los valores de respeto, participación e interacción que caracterizan una sociedad diversa cohesionada.

Consecuentemente y atendiendo a lo anteriormente expuesto, podríamos definir una buena práctica intercultural con los siguientes parámetros, identificados en MKC:

- Tiene un impacto tangible en la mejora de la calidad de vida de las personas

- Es sostenible social, cultural y económicamente, proporcionando beneficios a largo plazo.

- Permite la transferibilidad; diversos aspectos pueden aplicarse a otros lugares o en otros sectores.

- Es eficaz y eficiente, establece claramente sus objetivos, hace un uso racional de los recursos disponibles y evalúa los resultados. 
- Promueve la colaboración entre lo público y lo privado y, entre actores diversos de la sociedad.

- Garantiza la lógica interdisciplinar e intersectorial, aprovechando conocimientos y experiencias de todas las disciplinas y de distintos sectores de la sociedad.

- Fomenta la participación a través de mecanismos de consultas.

- Genera consenso, implicando a la mayoría de los ciudadanos.

- Supone la evaluación continua, determinando la consecución de los objetivos y la utilización racional de los recursos.

\subsection{Retos y desafios}

Varios son los retos y desafíos a los que se enfrenta el centro de Botkyrka. De ellos, los profesionales de la institución entrevistados para el estudio coinciden en tres puntos relevantes:

- $\quad$ Reducir la marginación de las minorías ${ }^{18}$

Las personas entrevistadas piensan que "la noción de interculturalidad entiende la diversidad como un recurso y, por ello, está asociada a la aceptación de los derechos humanos además de la conformidad de crear posibilidades igualitarias a las personas. Debemos trabajar con justicia social, creando las condiciones necesarias para que las personas puedan participar en los procesos democráticos de la sociedad de forma equitativa" (persona entrevistada 1). De esta forma, los factores principales para reducir la marginación son el bienestar comunitario, la participación cívica, el empleo y la educación. Aunque la participación en el mercado de trabajo es alta entre los suecos, disminuye entre los que tienen una educación mínima. El compromiso cívico es fuerte, pero se puede mejorar aún más asegurando que los residentes puedan plantear sus preocupaciones y lograr un impacto real en las políticas y servicios locales. De esta forma, el compromiso se volvería más incluyente y fomentaría la participación de los inmigrantes y los jóvenes. La salud, la vivienda, la seguridad, las actividades comunitarias y los espacios públicos acogedores ayudan a fortalecer las comunidades y permitir a los residentes asumir los retos de la educación, el desarrollo de habilidades y el compromiso cívico que reducen la marginación. Sin embargo, asegurar el acceso al mercado de trabajo para todos los residentes

\footnotetext{
${ }^{18}$ En relación con la definición de minorías en este caso, ver nota 12.
} 
es clave para reducir y prevenir la marginación. Aumentar la baja tasa de participación de mercado entre las personas de origen inmigrante reducirá la marginación, pero también contribuirá a una nueva identidad que reconozca la diversidad cambiando las percepciones entre la mayoría de los suecos. Además de las buenas prácticas actuales, como el empleo juvenil de verano y una fuerza laboral municipal diversa, deben incorporarse nuevas vías.

- Trabajar previniendo conflictos, con especial atención en los nuevos discursos políticos negativos enfocados a los nacionalismos exacerbados.

El trabajo interdisciplinar de MKC concentra sus esfuerzos en resolver aquellos conflictos que tienen su origen en el desconocimiento o en una falta de entendimiento. Previniendo la formación, proliferación y consolidación de estereotipos y prejuicios, rumores y percepciones negativas de la población e incluso, en los discursos políticos claramente construidos sobre la base de la diferencia negativa. Desde este último punto de vista, podríamos ver como ciertos discursos negativos penetran en la sociedad por una falta de afianzamiento de la interculturalidad, "actualmente, hay una retórica o un discurso nacionalista muy fuerte que se está desarrollando en Europa y a nivel internacional $\mathrm{y}$, donde las características de la sociedad se formulan en otras palabras ${ }^{19}$. Ideas que no favorecen la diversidad cultural ni tampoco el diálogo intercultural" (persona entrevistada 2). Por otro lado es importante destacar que hacer visible la estrategia de la interculturalidad de una forma demasiado rápida, como apuesta política sin aún haber penetrado en la sociedad y creando cohesión social ante la diversidad, igualmente pudiera producir un efecto negativo y ser la base para discursos xenófobos que pueden ser usados ante la población autóctona sin cultura en la diversidad. Consecuentemente es importante continuar con la gestión de la diversidad y con la implementación de las

\footnotetext{
${ }^{19}$ Los populismos han vivido un resurgimiento en las elecciones de las democracias occidentales gracias especialmente a los prejuicios arraigados entre algunos sectores de la población y a las crisis de refugiados en Europa. Aunque durante un tiempo el populismo fue un fenómeno marginal, no más del $10 \%$ del electorado, en diversos países europeos esta tendencia ha ido cambiando considerablemente en los últimos años. En el caso de Suecia, el partido de los Demócratas Suecos (SD) entró por primera vez en el Parlamento sueco en el año 2010 con un 5,7\% de los votos y 20 escaños, y en el año 2014 aumentaron su representación hasta el 12,9\%, consiguiendo 49 escaños. En las últimas elecciones celebradas en septiembre del año 2018, obtuvieron un $17,7 \%$ de representación, con 63 escaños. Los votantes de este partido ven a los inmigrantes como una amenaza para su bienestar económico, su sensación de seguridad y su identidad. Sienten que la influencia de otras culturas está diluyendo la identidad sueca, cambiando los valores y el modo de vida suecos.
} 
competencias interculturales de manera progresiva y afianzando un consenso en las administraciones públicas y en las organizaciones de la sociedad civil.

- Continuar la renovación de las instituciones suecas

Si bien se han producido importantes avances en la gobernanza local que desarrolla un proceso favorable hacia la interculturalidad, la renovación de las instituciones aún está incompleta y es conveniente extenderla hacia otras instituciones vitales, como son por ejemplo los museos, los teatros, los partidos políticos o las escuelas. "La gran mayoría de las instituciones suecas están desconectadas de la realidad de la sociedad, desconocen quiénes son sus gentes. Hay una importante necesidad de renovación entre las instituciones centrales de la democracia sueca" (persona entrevistada 3). La acomodación de la diversidad implica una política de reconocimiento y de respeto de la diferencia, asumiendo que estamos en un proceso de transformación donde todas las partes implicadas en la diversidad aceptan mutuamente contribuir en la construcción de un proyecto político cívico compartido. Es importante subrayar, sin embargo, que la acomodación de la diversidad no significa destruir aquello que siempre ha existido - la tradición-, sino construir entre todas las expresiones de la diversidad una nueva cultura cívica apoyada en la diversidad como valor.

\section{Conclusiones}

Si algo caracteriza a los temas relacionados con la gestión de la diversidad es su complejidad. Este aspecto influye claramente en el debate sobre qué tipo de liderazgo político es necesario ejercer. Apostar por la diversidad cultural y por la interculturalidad exige estimular el diálogo escuchando todas las opiniones, defender y transmitir unas ideas y unos principios claros y comprensibles. Desarrollar una pedagogía que explique los objetivos que persiguen las políticas que las impulsan, tejer complicidades y consensos tanto políticos como sociales, definir claramente cuál es el marco general en que se definen estas políticas y las líneas que no se deben cruzar. Dar a conocer realidades poco mediáticas pero que son claves para demostrar prejuicios y falsas percepciones, y no esconder realidades por complejas que sean, actuando para solucionarlas. De este modo, la interculturalidad exige, para avanzar hacia ella, contar con un liderazgo político proactivo, convencido y convincente que sea consciente de la dimensión del reto que afrontamos y con capacidad no sólo para el liderazgo de las políticas sino también para el debate público. 
En relación a cuáles son las políticas culturales del municipio de Botkyrka en un contexto basado en la diversidad cultural y el diálogo intercultural, y tras los resultados expuestos, una de las políticas culturales desarrolladas en el territorio está focalizada en la fundación Mångkulturellt centrum. Desde su creación en el año 1987, la institución está dirigida por un comité compuesto por personas procedentes de todos los partidos políticos del municipio de Botkyrka, una persona de la Universidad de Sódertörn (Södertörns högskola) y una persona perteneciente al Museo Nórdico (Nordiska Museet), museo dedicado a la historia y la cultura del pueblo sueco desde finales de la Edad media y hasta nuestros días. Mångkulturellt centrum funciona como lugar de encuentro y foro para diferentes formas de expresión, investigación, conocimientos y experiencias sobre la migración, la diversidad social y cultural. Las actividades desarrolladas conducen a mejoras sociales de forma activa, a un mayor conocimiento y a un aumento de las habilidades de las personas y las organizaciones, facilitando una sociedad sostenible. El éxito de la labor desempeñada hasta nuestros días, cumpliendo más de treinta años de existencia y siendo uno de los centros pioneros de Europa, lo atestigua el reconocimiento de su labor como buena práctica dentro del programa Ciudades Interculturales (Intercultural Cities) del Consejo de Europa, en el año 2016. Asimismo, Mångkulturellt centrum continúa trabajando por una sociedad donde la diversidad se refleja en la autoimagen nacional de Suecia. Una sociedad en la que nadie tiene que sacrificar su propia cultura e identidad con el fin de lograr una participación e integración. De esta forma afirmamos que la organización mejora la diversidad cultural y el diálogo intercultural en el territorio como así lo reflejan la visión y la misión del centro, las acciones generales desarrolladas a lo largo de los años teniendo como base sus cuatro objetivos principales. Destacando su capacidad de adaptación a los fenómenos cambiantes de la sociedad desde su creación y hasta nuestros días.

A propósito de las acciones concretas realizadas que mejoren la diversidad cultural y el diálogo intercultural, hemos constatado como una de las finalidades prioritarias de Mångkulturellt centrum es que los resultados obtenidos puedan ser utilizados en el trabajo práctico cotidiano, mejorando la vida de los grupos potenciales. Así, los proyectos culturales analizados por su singularidad, First Generation y Att minnas migrationen, muestran como la cultura es el medio a través del cual comunicamos lo que somos, lo que es importante para nosotros, lo que nos ha formado $\mathrm{y}$ que aspectos de nosotros mismos mantenemos a medida que avanzamos hacia el futuro. La identidad se define a menudo en términos culturales, al igual que la alteridad. 
Por lo tanto, es necesario y natural contemplar el ámbito de la cultura y de las artes cuando existe la necesidad de conocer a "los otros", con el objetivo de formar una sociedad inclusiva que pueda aprender a beneficiarse de la diversidad. Y en este sentido, las artes y la cultura poseen un potencial destacado que favorece la diversidad demográfica y la integración de las personas procedentes de otros países.

En relación a las conclusiones obtenidas sobre la identificación de los desafíos a los que se enfrenta Mångkulturellt centrum, y por ende y en gran medida las políticas locales del municipio de Botkyrka, las ideas aportadas por los expertos de la Fundación nos transmiten tres temas relevantes: la reducción de la marginación de las minorías, fomentando el bienestar comunitario, la participación cívica, el empleo y la educación; la prevención de conflictos, con especial atención en los nuevos discursos políticos negativos y enfocados a los nacionalismos exacerbados y; la continua renovación de las instituciones suecas, contribuyendo en la construcción de un proyecto político cívico compartido.

Al tenor de lo expuesto en las conclusiones podemos afirmar que el diálogo intercultural presupone la diversidad cultural y ofrece un instrumento eficaz para conectar a las personas con diferentes orígenes, intereses y perspectivas. Es una forma de implicar a los migrantes y refugiados del territorio, aumentando su participación en la vida cultural y social. Sin embargo, detectamos que la realización del diálogo intercultural en las prácticas cotidianas es un desafío en sí mismo y, exige cambios en las rutinas y procedimientos establecidos que normalmente pueden bloquearlo. Así, al examinar el diálogo intercultural, descubrimos como las políticas culturales, las artes y las prácticas culturales pueden contribuir a abordar este importante reto.

A través del presente estudio corroboramos como Mångkulturellt centrum es un buen ejemplo de la importancia de tener instituciones y organizaciones públicas que logren competencias interculturales, se abran al diálogo intercultural y trabajen junto con los propios inmigrantes y otros actores. Las culturas y las identidades -los elementos básicos del diálogo intercultural- no son nunca esencias fijas sino procesos fluidos y abiertos a transformaciones en las relaciones cotidianas entre personas de diferentes orígenes. Los individuos son moldeados y restructurados por sus relaciones con los demás, así como las relaciones son moldeadas y remodeladas por los propios individuos. Los proyectos y prácticas inspirados en el diálogo intercultural tienen el potencial de producir valores nuevos e inclusivos. 
Aun cuando los debates sobre el replanteamiento del diseño de las nuevas políticas culturales son vigentes, en el estudio del caso de Mångkulturellt centrum hemos detectado los objetivos principales que son aconsejables que éstas reúnan:

- $\quad$ Fomento de políticas innovadoras que promuevan las formas de la diversidad cultural.

- Ampliar el espacio democrático para que abarque la diversidad y la interdependencia en la construcción de la ciudadanía en los planos nacional e internacional.

- $\quad$ Prestar apoyo al pensamiento creador y a la investigación innovadora; prestar apoyo a las políticas locales, regionales y mundiales encaminadas al desarrollo para el bienestar en las que se establezca un vínculo en cuatro pilares o dimensiones.

- Mejorar la cooperación internacional.

El presente trabajo plantea un estudio parcial de las políticas culturales enfocadas a la diversidad cultural y el diálogo intercultural. Este tema aglutina gran número de aspectos que deben ser estudiados con más profundidad y que representan un importante reto para los investigadores actuales. Son necesarios un mayor conocimiento y un análisis exhaustivo de la diversidad cultural en todas sus facetas, tratando de exponer la complejidad del proceso y, al mismo tiempo, definir el rasgo común entre la amplia gama de interpretaciones posibles. Son imprescindibles estudios que persuadan a los encargados de adoptar decisiones y a las distintas partes interesadas de la importancia de invertir en la diversidad cultural en cuanto dimensión esencial del diálogo intercultural. Son necesarios el compromiso político y el consenso que garanticen la implementación de políticas relacionadas con la integración a largo plazo. Por otro lado, es necesario un análisis crítico de las prácticas y actividades desarrolladas por la administración, desde el punto de vista estratégico y operativo. Todo ello permitirá renovar, en una parte importante, nuestros enfoques del desarrollo sostenible, garantizar el ejercicio eficaz de las libertades y de los derechos humanos universalmente reconocidos y, fortalecer la cohesión social y la gobernanza democrática.

\section{Referencias}

Barreiro, B. (2011). La diversidad cultural en el derecho internacional: la Convención de la UNESCO. Madrid, España: Iustel Publicaciones. 
Bennett, T. (2001). Differing diversities. Transversal study on the theme of cultural policy and cultural diversity. Estrasburgo, Francia: Consejo de Europa.

Botkyrka Kommun. (2014). Facts about Botkyrka - context, character and demographics. Estocolmo, Suecia: Botkyrka Kommun.

Boveri, K. (2013). The political dinamics of multiculturalism in Sweden. En R. Taras (ed.), Challenging Multiculturalism: European Models of Diversity, 138-160. Edinburgh: Edinburgh University Press.

CGLEU, Ciudades y Gobiernos Locales Unidos. (2015). Agenda 21 de la Cultura. Acciones. Barcelona, España: CGLU.

CGLEU, Ciudades y Gobiernos Locales Unidos. (2009). Cultura y desarrollo sostenible: ejemplos de innovación institucional y propuesta de un nuevo modelo de política cultural. Resumen ejecutivo. Barcelona, España: CGLU.

Council of Europe. (2018). Intercultural cities programme. Obtenido de https://www.coe.int/en/web/interculturalcities/index-results-per-city

Daun, Å., Ehn, B., y Klein, B. (1982). To make the world safe for diversity. Towards and understanding of multi-cultural societies. Helsingborg, Suecia: The Swedish inmigration Institute and Museum.

Duxbury, N. (2008). Under construction. The state of cultural infraestructure in Canada. Vancouver, Canadá: Simon Frase University.

Duxbury, N., Cullen, C., y Pascual, J. (2012). Cities, culture and sustainable development. En H. Anheier, y Y. A. Isar, Cultural Policy and Governance in a New Metropolitan Age, 73-86. Londres, Reino Unido: Sage.

ECAR, European Cities Against Racism. (2013). Buenas prácticas. Modelos locales de lucha contra el racismo. Proyecto ECAR, ciudades europeas contra el racismo. Berlín, Alemania: ECAR.

Hawkes, J. (2001). The fourth pillar of sustainability. Culture's essential role in public planning. Melbourne, Australia: University Press.

Hosseini-Kaladjahi, H. (2002). Stora fiskar äter fortfarande små fiskar. Helhetsutvärdering av storstadssatsningen $i$ Botkyrka kommun. Estocolmo, Suecia: Mångkulturellt centrum.

León, R. (2011). Om behovet att utveckla indikatorer för ett kommunalt arbete mot diskriminering i Botkyrka. Estocolmo, Suecia: Mångkulturellt centrum. 
Magnusson, L. (2006). Exhibitions that investigate contemporary society. En M. Morberg (ed)., Arts ans diversity. Policies, research and practice, 55-62. Estocolmo, Suecia: Mångkulturellt centrum.

Mångkulturellt centrum - MKC (2006, 2008-2014, 2016-2018). Mångkulturellt centrum verksamhetsberättelse. Estocolmo, Suecia: Mångkulturellt centrum.

OEI, Organización de Estados Iberoamericanos. (2018). Cultura y desarrollo. Obtenido de HYPERLINK "http://www.oei.es/historico/cultura/cultura_desarrollo.htm" http://www.oei.es/historico/cultura/cultura_desarrollo.htm

Open Society Foundation. (2014). Europe's white working class communities. Stockholm. Nueva York, Estados Unidos: Open Society Foundation.

Pascual i Ruiz, J. (2007). Sobre la participación ciudadana en el desarrollo de politicas culturales locales en ciudades europeas. En Interarts, Guía para la participación ciudadana en el desarrollo de politicas culturales locales para ciudades europea, 10-41. Barcelona, España: Interarts / ECUMEST / Fundación Europea de la Cultura.

Pinyol, G. (2013). La política intercultural: una política de buenas prácticas. En R. Z.-B. (eds.), Manual para el diseño de politicas interculturales, 159-193. Barcelona, España: GRITIM-UPF.

Prieto, J., y Velasco, H.M. (2016). La diversidad cultural. Análisis sistemático e interdisciplinar de la Convención de la UNESCO. Madrid, España: Trotta Editorial.

PNUD, Programa de las Naciones Unidas para el Desarrollo. (2004). La libertad cultural en el munco diverso de hoy. Madrid, España: Ediciones Mundi-Prensa.

Robins, K. (2006). The challenges of transcultural diversities. Transversal study on the theme of cultural policy and cultural diversity: final report. Estrasburgo, Francia: Consejo de Europa.

Rojas, H. (2010). Strategy for an intercultural Botkyrka. Estocolmo, Suecia: Botkyrka Kommun.

Roth, H. I. y Hertzberg, F. (2012). Sweden. En R. Z.-B. (eds.), Addressing tolerance and diversity discourses in Europe, 103-124. Barcelona, España: CIDOBGRITIM-UPF.

SALAR, Swedish Association of Local Authorities and Regions. (2009). Culture in the sustainable society. Estocolmo, Suecia: SKL FS Grafisk Produktion. 
Tawat, M. (2014) Danish and Swedish inmigrants' cultural policies between 1960 and 2006: toleration and the celebration of difference. International Journal of Cultural Policy, 20(2), 202-220.

Tawat, M. (2017). The birth of Sweden's multicultural policy. The impact of Olof Palme and his ideas. Journal of Cultural Policy. https://doi.org/10.1080/10286632.2017.1335719

UNESCO. (2009). UNESCO Word report. Investing in Cultural diversity and intercultural dialogue. París, Francia: UNESCO.

UNESCO. (2010). Mapping cultural diversity. Good practices from around the globe. Bonn, Alemania: UNESCO.

UNESCO. (2011). Una nueva agenda de politicas culturales para el desarrollo y la comprensión mutua. París, Francia: UNESCO.

UNESCO. (2012). Textos fundamentales de la Convención de 2005 sobre la Protección y la Promoción de la Diversidad de las Expresiones Culturales. Paris, Francia: UNESCO.

UNESCO. (2018a). Declaración Universal de la UNESCO sobre la Diversidad Cultural. 2 de noviembre de 2011. Obtenido de UNESCO: http://portal.unesco.org/es/ev.php-

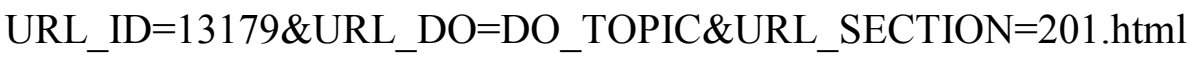

UNESCO. (2018b). Convención sobre la protección y la promoción de la diversidad de las expresiones culturales 2005. 20 de noviembre de 2005. Obtenido de UNESCO: http://portal.unesco.org/es/ev.phpURL_ID $=31038 \& U R L \_D O=D O \_T O P I C \& U R L \_S E C T I O N=201 . h t m l$

UNESCO. (2018c). Reflection on Cultural Diversity. Obtenido de UNESCO: http://www.unesco.org/new/es/culture/themes/cultural-diversity/reflections-oncultural-diversity/

Usallán, L. (2012). Diseño de la gestión de la diversidad cultural en entidades subestatales culturalmente diferenciadas. Un análisis sobre Quebec, Cataluña, País Vasco y País de Gales. Tesis Doctoral. Bilbao, España: Universidad de Deusto. 